\title{
Factors associated with medication nonadherence among Medicare low-income subsidy beneficiaries with diabetes, hypertension, and/or heart failure
}

\author{
Chi Chun Steve Tsang, PhD; Jamie Browning, PharmD/MBA Candidate; Lorraine Todor, PharmD Candidate; \\ Samantha Dougherty, PhD; Kenneth C Hohmeier, PharmD; Minghui Sam Li, PhD; Nancy Borja-Hart, PharmD, BCPS; \\ Lisa E Hines, PharmD, CPHQ; and Junling Wang, PhD
}

\section{What is already known about this subject}

- The low-income subsidy (LIS) program was developed to offer financial assistance to Medicare beneficiaries with low income and assets by reducing their premiums and/or copayments.

- Previous studies have identified factors associated with medication adherence and nonadherence, such as age, sex, race/ethnicity, and socioeconomic characteristics.

- Less is known about the factors associated with medication nonadherence, specifically in the Medicare LIS population.

\section{What this study adds}

- Individual and community characteristics are associated with the likelihood of medication nonadherence among Medicare LIS recipients with type 2 diabetes, hypertension, or heart failure.

- The following factors are positively associated with medication nonadherence: male sex, younger age, racial/ethnic minority status, living in a metropolitan statistical area or health professional shortage area, living in West or South regions, and a higher risk adjustment summary score.

- This study provided insight into medication nonadherence within the Medicare LIS population and identified the need to consider these factors when developing future policies to improve medication adherence.

\begin{abstract}
Author affiliations
Chi Chun Steve Tsang, PhD, Postdoctoral Fellow, Health Outcomes and Policy Research; Minghui Sam Li, PhD; and Junling Wang, PhD, Department of Clinical Pharmacy and Translational Science, University of Tennessee Health Science Center College of Pharmacy, Memphis. Jamie Browning, PharmD/MBA Candidate, and Lorraine Todor, PharmD Candidate, University of Tennessee Health Science Center College of Pharmacy, Memphis. Samantha Dougherty, PhD, Policy and Research, Pharmaceutical Research and Manufacturers of America (PhRMA), Washington, DC. Kenneth C Hohmeier, PharmD, and Nancy Borja-Hart, PharmD, BCPS, Department of Clinical Pharmacy and Translational Science, University of Tennessee Health Science Center College of Pharmacy, Nashville. Lisa E Hines, PharmD, CPHQ, Pharmacy Quality Alliance, Alexandria, VA.
\end{abstract}

AUTHOR CORRESPONDENCE: Junling Wang, 901.448.3601; jwang26@uthsc.edu 


\section{ABSTRACT}

BACKGROUND: Previous studies have documented factors influencing medication nonadherence among the Medicare population, but few studies have examined medication nonadherence among the Medicare low-income subsidy (LIS) population. Furthermore, little is known about the factors associated with nonadherence among this population, especially those with prevalent chronic conditions such as type 2 diabetes, hypertension, or heart failure.

OBJECTIVE: To examine factors associated with the likelihood of medication nonadherence among Medicare LIS recipients with type 2 diabetes, hypertension, or heart failure.

METHODS: This was a retrospective analysis of 2012-2013 Medicare Parts A, B, and D claims (most recent available for this research) linked to the Area Health Resources Files. Beneficiaries aged 65 years or older with continuous Medicare coverage and receiving any LIS were included. Individuals were categorized into full LIS or partial LIS groups. Nonadherence was determined by the proportion of days covered less than $80 \%$ for specified oral type 2 diabetes, hypertension, and heart failure medications, as defined by the Pharmacy Quality Alliance. A multivariate logistic regression was used to determine and compare individual-level and community-level characteristics associated with nonadherence among the entire study sample, the full LIS group, and the partial LIS group.

RESULTS: The study sample included 505,771 Medicare beneficiaries, with 448,509 (88.7\%) receiving full LIS and 57,262 (11.3\%) receiving partial LIS. The proportion of individuals nonadherent was higher among the full LIS population (33.2\%) than that of the partial LIS population (30.8\%). Among the entire population, younger age was associated with nonadherence $(\mathrm{OR}=0.98 ; 95 \% \mathrm{Cl}=0.98-0.99)$. Men were more likely to be nonadherent than women ( $\mathrm{OR}=1.12 ; 95 \%$ $\mathrm{Cl}=1.11-1.14$ ). Compared with non-Hispanic Whites, racial/ethnic minorities had higher nonadherence. Compared with beneficiaries who were non-Hispanic White, the ORs for those who were Black, Hispanic, Asian, and other were 1.41 (95\% Cl=1.38-1.43), 1.58 (95\% $\mathrm{Cl}=1.55-1.61), 1.08$ (95\% Cl=1.05-1.11), and $1.63(95 \% \mathrm{Cl}=1.56-1.70)$, respectively. There were higher nonadherence rates among patients living in communities with lower socioeconomic characteristics, such as a metropolitan statistical area (MSA vs non-MSA; OR $=1.05,95 \%$ $\mathrm{Cl}=1.04-1.07)$. A higher risk adjustment summary score, indicating worse health status, was associated with an increased likelihood of medication nonadherence $(\mathrm{OR}=1.21 ; 95 \% \mathrm{Cl}=1.20-1.22)$. These patterns were similar among the full and partial LIS groups.

CONCLUSIONS: Individual- and community-level characteristics were associated with the likelihood of medication nonadherence among Medicare LIS recipients with type 2 diabetes, hypertension, or heart failure. These characteristics included younger age, male sex, racial/ ethnic minorities, living in lower socioeconomic communities, and a higher risk adjustment summary score. This study provided insight into medication nonadherence within the Medicare LIS population and identified the need to consider these factors when developing future policies to improve medication adherence.
The Centers for Medicare \& Medicaid Services (CMS) established the Medicare prescription drug (Part D) benefit based on the Medicare Prescription Drug, Improvement, and Modernization Act in 2006. ${ }^{1}$ Medicare is a federal program providing health insurance for individuals aged at least 65 years and those with specific disabilities. Part D represents a landmark improvement to the Medicare program, and studies have demonstrated the benefits of this program in reducing out-of-pocket costs of medication use, improving medication use, reducing hospitalization and emergency department visits, and reducing racial/ethnic disparities in medication use. ${ }^{1-4}$ To further reduce the burden of prescription medication cost among individuals with limited financial resources, CMS also provides lowincome subsidies (LIS) for individuals with limited income and resources. ${ }^{1,5}$

The LIS program offers financial assistance that reduces the premiums and/or copayments/coinsurance for Medicare LIS beneficiaries. These beneficiaries receive full or partial LIS with different subsidy levels depending on insurance status and financial resources. ${ }^{6}$ In addition to Medicare coverage, LIS-eligible beneficiaries receive this additional financial assistance through Medicaid, a state-managed health insurance program for specific categories of low-income individuals. To be eligible for full LIS, patients must be eligible for their state Medicaid programs or have an annual income below $135 \%$ of the federal poverty level (FPL). To be eligible for partial LIS, patients must have an annual income below $150 \%$ of the FPL. ${ }^{6}$ For individuals receiving full LIS, the copayment for Part D formulary drugs ranges from zero to $\$ 3.60$ for generic drugs and is $\$ 8.95$ for brand name drugs. Individuals receiving partial LIS pay \$3.60 for generic drugs, \$8.95 for formulary medications, and a $15 \%$ copay for the catastrophic coverage.

The LIS program has reduced prescription drug costs, improved medication use, and reduced racial/ethnic disparities in medication use for the recipients of an LIS. For example, according to the analysis by Chou et al, among individuals with non-small cell lung cancer, patients with no or partial LIS had a $23 \%$ lower likelihood of initiating oral anticancer treatments compared with patients with full LIS. ${ }^{7}$ Biggers et al studied disparities in persistence and adherence to breast cancer hormonal therapy and found racial/ethnic disparities in persistence and adherence to medications among unsubsidized individuals but not present among subsidized individuals. ${ }^{8}$ For example, unsubsidized Black women had 109\% higher hazards of discontinuation than their subsidized counterparts. ${ }^{8}$

Significant literature has documented factors associated with medication adherence and nonadherence. However, this has not been adequately examined among the LIS 
population, particularly among individuals with prevalent chronic conditions. There are significant systematic differences between the LIS and the non-LIS populations. The LIS population has lower socioeconomic characteristics than the non-LIS population. ${ }^{1}$ The LIS population has more comorbidities with a higher prevalence of type 2 diabetes, congestive heart failure, peripheral vascular diseases, and dementia than the non-LIS population. The LIS population also incurs higher overall health care expenditures and pays lower out-of-pocket expenditures. ${ }^{9}$ Patients in the LIS population live in communities where individuals belong to lower socioeconomic categories. The LIS population also fills more prescription medications than the non-LIS population. ${ }^{9}$ Analyses of medication adherence among the LIS population compared with the non-LIS population have been inconsistent. ${ }^{9,10}$

The objective of this study was to determine the factors associated with medication nonadherence among Medicare LIS beneficiaries with type 2 diabetes, hypertension, and heart failure. These are the most prevalent health conditions among the Medicare population. ${ }^{11}$ This analysis can help clinical and policy decision makers identify individuals at a higher risk of medication nonadherence and target interventions to improve medication adherence.

\section{Methods}

This retrospective study analyzed Medicare Parts A, B, and D data linked to the Master Beneficiary Summary File (MBSF) from 2012 to 2013 and Area Health Resources Files (AHRF). ${ }^{12,13}$ Medicare Parts A and B provided diagnostic records of patients from inpatient and outpatient services. Medicare Part D data provided information on patient medication use, such as service date, National Drug Code numbers, quantity dispensed, days supply, cost, and payment. The MBSF offered demographic and enrollment information for Medicare beneficiaries. Medicare data from 2012-2013 were the most recent data available for this research. Because of limited demographic information in the MBSF, the AHRF was used to acquire population and community characteristics at the county level. AHRF is a free federal database managed by the Health Resources \& Services Administration, a federal agency for improving access to health care services for vulnerable populations..$^{13}$

The theoretical framework of this study was the GelbergAndersen Behavioral Model for Vulnerable Populations. ${ }^{14}$ This framework was selected because the study outcome was the use of prescription medications. ${ }^{14}$ Based on this framework, variables in regression models were categorized into predisposing, enabling, and need factors.
Predisposing factors "predispose" people to the use of medications. These variables included 3 individual-level characteristics (age, sex, and race/ethnicity) and 4 community-level characteristics (percentage of married-couple families, per capita income, percentage of people without health insurance, and percentage of people aged 25 years and older with education levels of less than high school level and at least high school level).

Enabling factors, which "enable" an individual's use of medications, included 3 community-level characteristics: metropolitan statistical area (MSA; yes/no), health professional shortage area (HPSA; yes/no), and census regions (Northeast, Midwest, South, West, and other).

Need factors measured patient need for prescription medications. This category included a risk adjustment summary score developed by CMS to adjust payments to Medicare Advantage plans based on patient clinical and demographic characteristics. ${ }^{15}$ Therefore, a higher score signifies worse health status. All individual-level factors were directly retrieved from Medicare data, and community-level factors were retrieved from AHRF.

Racial/ethnic groups included were non-Hispanic White, Black, Hispanic, Asian/Pacific Islander (Asian), and others (including American Indian/Alaska Native, other, and unknown). Medicare data are reasonably valid for race/ethnicity for the 4 individual racial/ethnic groups just named but not for American Indian/Alaska Native. ${ }^{16}$ Therefore, those who were American Indian/Alaska Native were combined with other and unknown race/ethnicity individuals.

To reduce population heterogeneity, the authors only included in the study sample patients aged 65 years or older (Figure 1). To obtain at least 1 year worth of data, patients needed to be alive during the entire study period. Only individuals with continuous Parts A, B, and D coverage were included to eliminate any coverage gap. Patients needed to have at least 1 disease of interest: type 2 diabetes, hypertension, or heart failure. Patients with at least 1 diagnostic claim for any of the conditions of interest were identified using the International Classification of Diseases, Ninth Revision, Clinical Modification diagnosis codes (Supplementary Table 1, available in online article). To be included in the nonadherence calculation, patients must have filled at least 2 prescriptions for the medications of interest and patients with type 2 diabetes could not be on insulin. All patients in the sample received LIS at some point during the study period. Patients were divided into 2 mutually exclusive cohorts: those with full LIS every month during the study period and those with partial LIS.

Eleven classes of medications were included in the analysis based on the technical specifications developed 


\section{FIGURE 1 Study Selection Process}

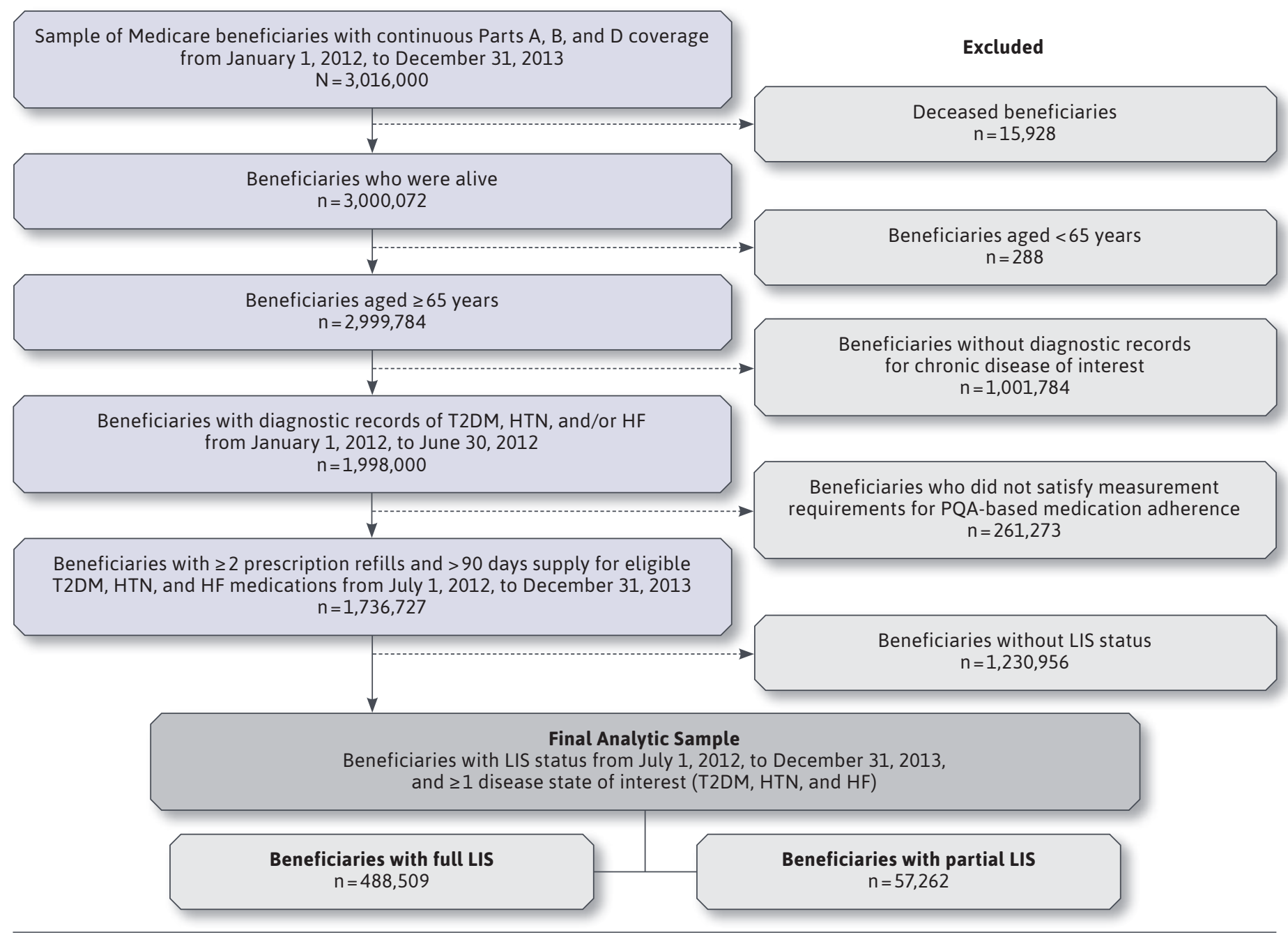

$\mathrm{HF}=$ heart failure; HTN=hypertension; LIS=low-income subsidy; PQA = Pharmacy Quality Alliance; T2DM=type 2 diabetes mellitus.

by the Pharmacy Quality Alliance (PQA). ${ }^{17} \mathrm{PQA}$, a non-profit organization established as a public-private partnership with CMS, was founded after the implementation of Medicare Part D with the purpose of developing performance measures to evaluate the safety and appropriateness of medication use. Oral medications for type 2 diabetes, hypertension, and heart failure were included in this study. Type 2 diabetes medications included biguanides, sulfonylureas, a-glucosidase inhibitors, thiazolidinediones, dipeptidyl peptidase-IV inhibitors, and incretin mimetics. Hypertension medications included beta blockers, calcium channel blockers, angiotensin II receptor blockers, angiotensin converting enzyme inhibitors, and direct renin inhibitors. The included heart failure medications were renin angiotensin system antagonists, beta blockers, and calcium channel blockers (data on file, Pharmacy Quality Alliance, Technical specifications for PQA-endorsed measures, 2018).

This study's medication adherence was measured by the proportion of days covered (PDC), and prescription claim records were used for the calculation based on PQA technical specifications. PDC was calculated at the condition level, and all medications for that condition were included in the PDC measure for a condition. For patients 
TABLE 1 Patient Characteristics in Study Population

\begin{tabular}{|c|c|c|c|c|c|c|}
\hline \multirow[b]{2}{*}{ Characteristics $^{\mathbf{a}}$} & \multicolumn{2}{|c|}{$\begin{array}{c}\text { All } \\
(\mathrm{N}=505,771)\end{array}$} & \multicolumn{2}{|c|}{$\begin{array}{c}\text { Full LIS } \\
(n=448,509,88.7 \%)\end{array}$} & \multicolumn{2}{|c|}{$\begin{array}{c}\text { Partial LIS } \\
\text { (n=57,262, 11.3\%) }\end{array}$} \\
\hline & $\mathbf{n}$ & $\%$ & $\mathbf{n}$ & $\%$ & $\mathbf{n}$ & $\%$ \\
\hline \multicolumn{7}{|l|}{ Predisposing factors } \\
\hline Age, mean (SD) & $78.3 \quad(7.8)$ & & $78.3 \quad(7.9)$ & & $78.1 \quad(7.5)$ & \\
\hline Male & 140,731 & 27.8 & 125,772 & 28.0 & 14,959 & 26.1 \\
\hline Female & 365,040 & 72.2 & 362,737 & 72.0 & 42,303 & 73.9 \\
\hline \multicolumn{7}{|l|}{ Race/ethnicity } \\
\hline Non-Hispanic White & 275,177 & 54.4 & 234,587 & 52.3 & 40,590 & 70.9 \\
\hline Black & 93,461 & 18.5 & 83,251 & 18.6 & 10,210 & 17.8 \\
\hline Hispanic & 83,079 & 16.4 & 78,380 & 17.5 & 4,699 & 8.2 \\
\hline Asian & 44,345 & 8.8 & 43,255 & 9.6 & 1,090 & 1.9 \\
\hline Other & 9,709 & 1.9 & 9,036 & 2.0 & 673 & 1.2 \\
\hline$\%$ married, mean $(S D)^{b}$ & $74.3 \quad(7.4)$ & & $74.1 \quad(7.5)$ & & $75.9 \quad(6.9)$ & \\
\hline \multicolumn{7}{|l|}{ Education among age $25+$ years $^{b}$} \\
\hline < High school, mean (SD) & $16.5 \quad(6.7)$ & & $16.7 \quad(6.8)$ & & $15.4 \quad(6.1)$ & \\
\hline z High school, mean (SD) & $83.6 \quad(6.7)$ & & $83.5 \quad(6.8)$ & & $84.7 \quad(6.1)$ & \\
\hline Per capita income (in $\$ 1,000$ ), mean $(S D)^{b}$ & $43.3(13.3)$ & & $43.6(13.6)$ & & $41.4(10.9)$ & \\
\hline$\%$ no insurance, mean (SD) ${ }^{\mathrm{b}}$ & $15.7 \quad(5.2)$ & & $15.8 \quad(5.3)$ & & $14.4 \quad(4.4)$ & \\
\hline \multicolumn{7}{|l|}{ Enabling factors } \\
\hline Metropolitan statistical area ${ }^{\mathrm{b}}$ & 390,095 & 77.1 & 349,529 & 77.9 & 40,566 & 70.8 \\
\hline Health professional shortage area $^{\mathrm{b}}$ & 463,999 & 91.7 & 413,420 & 92.2 & 50,579 & 88.3 \\
\hline \multicolumn{7}{|l|}{ Census regions $^{\mathrm{b}}$} \\
\hline Northeast & 102,702 & 20.3 & 93,269 & 20.8 & 9,433 & 16.5 \\
\hline Midwest & 87,986 & 17.4 & 74,959 & 16.7 & 13,027 & 22.8 \\
\hline South & 218,044 & 43.1 & 188,808 & 42.1 & 29,236 & 51.1 \\
\hline West & 96,417 & 19.1 & 90,910 & 20.3 & 5,507 & 9.6 \\
\hline Other & 622 & 0.1 & 563 & 0.1 & 59 & 0.1 \\
\hline \multicolumn{7}{|l|}{ Need factor } \\
\hline Risk adjustment summary score, mean (SD) & $1.3(0.8)$ & & $1.4(0.8)$ & & $1.0(0.7)$ & \\
\hline \multicolumn{7}{|c|}{$\begin{array}{l}\text { Note: All characteristics were significantly different between individuals with full LIS and partial LIS with P<0.05. } \\
{ }^{a} \text { Number and frequency unless otherwise specified. } \\
{ }^{b} \text { Community-level factor. } \\
\text { LIS=low-income subsidy. }\end{array}$} \\
\hline
\end{tabular}

with 1 chronic condition, PDC of at least $80 \%$ for all study medications for that condition was considered adherent. Patients were considered adherent only if PDC was at least $80 \%$ for medications for each disease of interest among individuals with multiple chronic conditions.

In the descriptive analysis, the characteristics of patients with full LIS and partial LIS were first compared. For categorical variables, proportions were calculated for each group, and a chi-square test was used to test the difference between groups. For continuous variables, means and SDs were calculated for each group, and a t-test was then used to compare between the groups. Bivariate analysis was then performed to determine the association between each factor and medication nonadherence.

Finally, in multivariate analysis, a "saturated" logistic regression model was used because the study outcome was 


\section{TABLE 2 Factors Associated with Nonadherence Based on Logistic Regression}

\begin{tabular}{|c|c|c|c|c|c|c|}
\hline & \multicolumn{2}{|c|}{ Overall } & \multicolumn{2}{|c|}{ Full LIS } & \multicolumn{2}{|c|}{ Partial LIS } \\
\hline & Odds Ratio & $95 \% \mathrm{Cl}$ & Odds Ratio & $95 \% \mathrm{Cl}$ & Odds Ratio & $95 \% \mathrm{Cl}$ \\
\hline \multicolumn{7}{|l|}{ Predisposing factors } \\
\hline Age & 0.98 & $0.98-0.99$ & 0.99 & $0.98-0.99$ & 0.98 & $0.98-0.99$ \\
\hline Female & - & - & - & - & - & - \\
\hline Male & 1.12 & $1.11-1.14$ & 1.12 & $1.10-1.14$ & 1.12 & $1.08-1.17$ \\
\hline Non-Hispanic White & - & - & - & - & - & - \\
\hline Black & 1.41 & $1.38-1.43$ & 1.40 & $1.38-1.43$ & 1.45 & $1.38-1.53$ \\
\hline Hispanic & 1.58 & $1.55-1.61$ & 1.58 & $1.55-1.61$ & 1.58 & $1.48-1.70$ \\
\hline Asians/Pacific Islander & 1.08 & $1.05-1.11$ & 1.08 & $1.05-1.11$ & 1.25 & $1.10-1.43$ \\
\hline Other & 1.63 & $1.56-1.70$ & 1.63 & $1.56-1.70$ & 1.62 & $1.39-1.90$ \\
\hline$\%$ married families in community ${ }^{a}$ & 1.26 & $1.14-1.40$ & 1.27 & $1.13-1.42$ & 1.05 & $0.75-1.46$ \\
\hline$\% \geq$ high schoola $^{a}$ & 1.65 & $1.39-1.96$ & 1.65 & $1.38-1.98$ & 1.22 & $0.73-2.02$ \\
\hline Per capita income (in $\$ 1,000)^{a}$ & 1.00 & $1.00-1.00$ & 1.00 & $1.00-1.00$ & 1.00 & $1.00-1.00$ \\
\hline$\%$ no insurance ${ }^{a}$ & 3.08 & $2.39-3.96$ & 2.95 & $2.25-3.86$ & 3.93 & $1.84-8.38$ \\
\hline \multicolumn{7}{|l|}{ Enabling factors } \\
\hline Non-metropolitan statistical area & - & - & - & - & - & - \\
\hline Metropolitan statistical area ${ }^{a}$ & 1.05 & $1.04-1.07$ & 1.05 & $1.03-1.07$ & 1.08 & $1.03-1.13$ \\
\hline Non-health professional shortage area ${ }^{a}$ & - & - & - & - & - & - \\
\hline Health professional shortage area ${ }^{a}$ & 1.04 & $1.01-1.06$ & 1.03 & $1.01-1.06$ & 1.04 & $0.98-1.11$ \\
\hline Northeast $^{\mathrm{a}}$ & - & - & - & - & - & - \\
\hline Midwest $^{a}$ & 1.09 & $1.06-1.11$ & 1.09 & $1.07-1.12$ & 1.04 & $0.98-1.11$ \\
\hline South ${ }^{\mathrm{a}}$ & 1.23 & $1.21-1.25$ & 1.24 & $1.22-1.27$ & 1.12 & $1.05-1.19$ \\
\hline West $^{a}$ & 1.23 & $1.20-1.26$ & 1.23 & $1.20-1.26$ & 1.26 & $1.16-1.37$ \\
\hline \multicolumn{7}{|l|}{ Need factor } \\
\hline Risk adjustment summary score & 1.21 & $1.20-1.22$ & 1.21 & $1.20-1.22$ & 1.26 & $1.23-1.29$ \\
\hline $\begin{array}{l}\text { Note: Reference groups are those with dashes. } \\
\text { variable. For instance, a 1-year increase in age } \\
\text { aCommunity-level factors. } \\
\text { LIS=low-income subsidy. }\end{array}$ & $\begin{array}{l}\text { ous variables } d \\
\text { ciated with a } 2\end{array}$ & $\begin{array}{l}\text { thave referer } \\
\text { wer odds of } k\end{array}$ & $\begin{array}{l}\text { groups. Their in } \\
\text { g nonadherent. }\end{array}$ & retation is for & nit of change in & quantity of $t$ \\
\hline
\end{tabular}

whether patients were nonadherent to medications or not. Odds ratios (ORs) were produced for categorical and continuous variables. Regarding the interpretation of ORs, for a categorical variable, an OR greater than 1 for a category suggests that the category is associated with higher odds of nonadherence compared with the reference group; for a continuous variable, an OR greater than 1 indicates that higher values of this variable are associated with higher odds of nonadherence.

Data analysis was conducted using SAS version 9.4 (SAS Institute). Probabilities less than 0.05 were considered statistically significant. This study was approved by the
Institutional Review Board (approval number 15-04242-XM) at the corresponding author's institution.

\section{Results}

As seen in Figure 1, this study included 505,771 Medicare LIS beneficiaries, of which 448,509 (88.7\%) received full LIS subsidy, and 57,262 (11.3\%) received partial LIS subsidy. The characteristics of the entire study population were analyzed (Table 1). Regarding the patient-level predisposing factors, the average (SD) age was 78.3 (7.8) years among the entire study population (those with full and partial LIS). 
Only 140,731 (27.8\%) individuals were male. Most of the study population were non-Hispanic White (54.4\%). The remaining were Black (18.5\%), Hispanic (16.4\%), Asian (8.8\%), and others (1.9\%). Concerning community-level predisposing factors, the average (SD) proportion of married-couple families was $74.3 \%$ (7.4\%); the average (SD) proportion of individuals with a high school or higher education in the community was $83.6 \%$ (6.7\%); and the average (SD) per capita income in $\$ 1,000$ was 43.3 (13.3). The average (SD) proportion of individuals without health insurance was $15.7 \%$ (5.2\%). With regards to enabling factors, most individuals lived in MSAs (77.1\%) and HPSAs (91.7\%). The highest percentage of the population lived in the South region (43.1\%), while the remainder lived in the Northeast (20.3\%), Midwest (17.4\%), West (19.1\%), and other regions (0.1\%). Regarding the need factor, the average (SD) risk adjustment summary score was $1.3(0.8)$.

Population characteristics were compared between the full LIS and partial LIS populations (Table 1). Differences in all characteristics of the people were statistically significant at $\mathrm{P}<0.05$. The full LIS population was slightly older than the partial LIS population (78.3 years vs 78.1 years), although this difference is not likely clinically insignificant. The full LIS population had a higher percentage of males (28.0\%) than the partial LIS population (26.1\%). The proportion of those who were non-Hispanic White was lower in the full LIS population (52.3\%) than the partial LIS population (70.9\%). Compared with the partial LIS population, the full LIS population had a lower proportion of married-couple families in the community (74.1\% vs $75.9 \%)$ and a lower proportion of individuals with an education level at or higher than high school in the community ( $83.5 \%$ vs $84.7 \%$ ).

Patients with full LIS lived in communities with higher per capita income (43.6 vs 41.4 , in $\$ 1,000$ ) and a higher percentage of people without health insurance (15.8\% vs $14.4 \%$ ). The full LIS population had higher rates living in MSAs (77.9\% vs $70.8 \%$ ) and HPSAs (92.2\% vs $88.3 \%$ ). Individuals with full LIS were more likely to reside in Northeast and West census regions than those with partial LIS. The full LIS population had a higher risk adjustment summary score of 1.4 compared with the partial LIS population risk adjustment summary score of 1.0.

The proportion of patients nonadherent to medications among the full LIS population (33.2\%) was higher than the partial LIS population (30.8\%). The difference of $2.4 \%$ in nonadherence $(\mathrm{P}<0.0001)$ between the 2 groups amounted to $7.79 \%$ of the rate among the partial LIS population. Multivariate analysis was conducted on factors associated with nonadherence among the entire population, those with full LIS, and those with partial LIS separately
(Table 2). Among the entire study population, younger age was associated with nonadherence. A 1-year increase in age was associated with a $2 \%$ lower odds of being nonadherent $(\mathrm{OR}=0.98,95 \% \mathrm{CI}=0.98-0.99)$. Male sex was positively associated with the likelihood of being nonadherent $(\mathrm{OR}=1.12$, 95\% CI $=1.11-1.14$ ).

Racial/ethnic minorities had a higher likelihood of nonadherence. Compared with those who were non-Hispanic White, the ORs for those who were Black, Hispanic, Asian, and other were 1.41 (95\% CI=1.38-1.43), 1.58 (95\% CI=1.551.61), 1.08 (95\% CI=1.05-1.11), and 1.63 (95\% CI=1.56-1.70), respectively. Higher proportions of married-couple families in the community were positively associated with nonadherence $(\mathrm{OR}=1.26,95 \% \mathrm{CI}=1.14-1.40)$, as was the percentage of the population with a high school or higher education $(\mathrm{OR}=1.65,95 \% \mathrm{CI}=1.39-1.96)$. The community's per capita income was not significantly associated with nonadherence $(\mathrm{OR}=1.00,95 \% \mathrm{CI}=1.00-1.00)$.

The percentage of the community's uninsured population was positively associated with nonadherence $(\mathrm{OR}=3.08$, $95 \% \mathrm{CI}=2.39-3.96)$. Individuals living in MSAs were more likely to be nonadherent than those in non-MSAs (OR=1.05, 95\% CI $=1.04-1.07)$, as were individuals living in HPSAs than those in non-HPSAs (OR=1.04, 95\% $\mathrm{CI}=1.01-1.06)$. Compared with individuals living in the Northeast, individuals from other regions had higher nonadherence with $\mathrm{OR}=1.09$ (95\% CI=1.06-1.11) for the Midwest, OR=1.23 (95\% CI=1.211.25) for South, and OR=1.23 (95\% CI=1.20-1.26) for West regions. The risk adjustment summary score was positively associated with nonadherence. A 1-unit increase in risk adjustment summary scores was associated with a $21 \%$ increase in patients' odds of being nonadherent $(\mathrm{OR}=1.21$, 95\% CI $=1.20-1.22$ ).

Based on the multivariate analysis among the full LIS population, patient/community characteristics associated with nonadherence were similar to the entire population. However, among those with partial LIS, some factors were nonsignificant: percentage of married-couple families in the community, percentage of people with an educational level of high school and above in the community, residing in an HPSA, and living in the Midwest region. Other factors had a similar significant association with nonadherence among the partial LIS population as the entire population.

\section{Discussion}

Using Medicare claims data, this study examined factors associated with nonadherence to medications for type 2 diabetes, hypertension, and heart failure among individuals with these conditions. Based on the multivariate 
analysis, all factors included in the regression based on the Gelberg-Andersen model were significant predictors of nonadherence except per capita income in the community. These factors included age (only factor negatively associated with nonadherence), male sex, race/ethnicity (minority status positively associated with nonadherence), percentage of married-couple families in the community, percentage of individuals with high school education or higher, percentage of people without health insurance, MSAs, HPSAs, census regions (Midwest, South, and West, all positively associated), and risk adjustment summary score. Based on the multivariate analysis, while all factors were significant predictors of nonadherence among the entire population and those with full LIS, some community factors were not significantly associated with nonadherence among the partial LIS population.

Medication adherence is critical because chronic conditions, such as those examined in this study, are predominantly managed by medications. Previous literature has generally documented that the use of prescription medications appropriately offsets health services costs. ${ }^{18-21}$ The Congressional Budget Office also acknowledges that increasing prescription use (prescriptions filled) by $1 \%$ can lower medical costs by $0.2 \%$ in the Medicare program. ${ }^{18}$ It also indicates that this offsetting effect of medications would be applied when estimating the future impact of policy proposals. Specifically, after evaluating a policy's direct impact on the number of prescription medications filled, the offsetting effects will be applied to the total budget. In addition, the literature also documents that medication nonadherence causes $10 \%$ of hospitalizations and increases health care costs by $\$ 100-\$ 290$ billion annually. ${ }^{19-21}$

The association between the factors and medication nonadherence found in this study was generally consistent with what has been reported in the literature despite the difference in study populations. Age has been shown to have mixed effects on medication nonadherence. ${ }^{10,22,23}$ This study found that older age was negatively associated with medication nonadherence, which is likely because younger individuals have a varied daily schedule and do not consider medication adherence a priority. ${ }^{10,23}$ Previously, male sex has been reported to be negatively associated with medication nonadherence, and it has been hypothesized that women may be more devoted to the care of other people instead of themselves. ${ }^{10,22}$ The contrasting finding in this study may be explained by the advanced age of the study population. With a higher percentage of women than men in the population, women in advanced age can focus on their health after their spouses are deceased.

There has been abundant literature on racial/ethnic disparities concerning access to health care in general and medication adherence. ${ }^{24}$ Such literature also stresses persistent racial/ethnic disparities despite efforts to address them. For example, based on a mid-decade assessment of Healthy People 2010, success in achieving its first goal of increasing quality and duration of healthy life showed limited concordance with its second goal of eliminating disparities. Despite progress in meeting the national targets for most of the objectives, relative disparities remained essentially the same for 157 of 195 objectives. ${ }^{25}$

The positive association between minority status and medication nonadherence among the study population in a subsidized health insurance program in Medicare is a sober reminder of stubborn racial/ethnic disparities. Based on the theory of fundamental causes, the relationship between patient socioeconomic status and health can be "reproduced" over time. ${ }^{26}$ This can happen because advantageous groups always command more resources and gain access to more opportunities than other groups. Therefore, to reduce health disparities, proponents of the theory of fundamental causes have suggested that interventions need to be applied to the entire population irrespective of individual status or behavior. ${ }^{26}$ To improve medication adherence among racial/ ethnic minorities, initiatives also need to benefit all patients regardless of patient characteristics and status.

Higher socioeconomic characteristics in the community are negatively correlated with medication nonadherence. Such association is not surprising, and this is consistent with the literature. ${ }^{27}$ Since community measures are not precise for an individual's socioeconomic status, such findings may stress the importance of the "nature versus nurture" discussion. This type of discussion centers around whether environmental factors in the community are significant predictors of patient behavior, such as medication nonadherence, independent of health care services patients receive. ${ }^{27}$ The risk adjustment summary score had a positive association with medication nonadherence. This may be because individuals with higher scores take more medications, which is generally associated with a lower level of medication adherence. ${ }^{22,23}$

Overall, the LIS program is a beneficial program for Medicare beneficiaries because of the high burden of health care costs among Medicare beneficiaries. According to a study by Cubanskil et al from the Kaiser Family Foundation, in 2013, 36\% of Medicare beneficiaries in traditional Medicare spent 20\% or more of their per capita total income on out-of-pocket health care costs. ${ }^{28}$ Among individuals aged 85 years and older, individuals with fair/ poor health, and population with low income, these proportions were $53 \%, 51 \%$, and $43 \%$, respectively. ${ }^{28}$

According to Fidelity Benefits Consulting, a couple in retirement needs to have access to $\$ 300,000$ in 2021 US 
dollars for out-of-pocket health care costs, excluding long-term care. ${ }^{29}$ This amount of money can easily overwhelm the resources of couples who on average have $\$ 90,000$ in their retirement account. ${ }^{30}$ Therefore, health care costs are a significant burden for people with Medicare, and programs such as LIS are much needed. However, even among individuals with LIS, medication nonadherence is still an issue. This study provides information on the target population that policymakers should keep in mind when designing programs to improve adherence.

This study's focus was medication use among Medicare beneficiaries, but prescription drug cost is only part of overall health care costs. Medicare beneficiaries still pay a significant amount of out-of-pocket costs for health care. For example, in 2014, among patients with income under $150 \%$ of the FPL, individuals spent over $25 \%$ of their income on premiums and medical care. ${ }^{31}$ This is partially because a significant number of people are not enrolled in programs such as the LIS program. For instance, Shoemaker et al reported that onethird of the Medicare beneficiaries potentially eligible for LIS did not enroll in the LIS program..$^{32}$ Therefore, proactive recruitment initiatives are needed to increase the enrollment of the population in such programs. ${ }^{32}$

While the Medicare Part D program and its LIS program have established benefits for Medicare beneficiaries, it has its share of challenges. For example, Decarolis analyzed Part D plans' behaviors in response to the different regulations regarding plan premiums for LIS recipients. ${ }^{33} \mathrm{He}$ found that insurers maximize their profit by a manipulative practice of clustering their premiums at a certain level. ${ }^{33}$ The LIS program has significant room for improvement before it fulfills its potential to help the Medicare population.

\section{LIMITATIONS}

Despite the contributions of this study to the literature, its findings should be interpreted and generalized with a few caveats. Because this study was an analysis of prescription fills, only filled prescriptions were included, but it was not determined whether patients actually took medications filled. More recent data than from 2012-2013 were not available, and disease-specific analysis was not conducted or reported. However, the consistency of the study results with previous research indicates the reliability and the robustness of the study findings.

This study used county-level community characteristics as proxies for patient socioeconomic characteristics because of the lack of such information at the patient level. Although AHRF has been extensively used for this purpose, this method may have introduced misclassification; however, the extent of this was difficult to calculate. Future studies should use individual-level patient characteristics to better understand the complex interactions between these characteristics and medication nonadherence. ${ }^{8}$

This analysis used data from the Medicare LIS population, so the results may not be generalizable to the commercial population. Because of the introduction of new medications every year and changes in medical practice, this study's patterns may be different from what would be found in data from more recent years. Since Part D data were compiled for payment purposes and not for research, some variables that describe critical patient characteristics, such as body mass index, smoking history, physical activities, and lab test results, were not available. Therefore, this analysis may involve omitted variable bias. ${ }^{34}$ Future studies including more detailed measurements may produce a more comprehensive answer to the research question.

\section{Conclusions}

Among the LIS population, patient and community characteristics are significant predictors of medication nonadherence. Significant factors for full LIS and partial LIS populations are younger age, male sex, racial/ethnic minority status, residing in communities with lower socioeconomic status, living in MSAs and HPSAs, living in the West and South census regions, and a higher risk adjustment summary score. These are not modifiable factors, but our findings can provide insight into the target population for decision makers who intend to improve medication adherence. Health care providers working with individuals with the characteristics mentioned in this study may also need extra support.

Future studies should determine whether medication nonadherence in the LIS population has resulted in higher consumption of health care resources, such as hospitalization and emergency department visits, compared with the non-LIS population. Future researchers should also refine strategies to enhance medication adherence among the LIS population. 


\section{DISCLOSURES}

This study was funded by the Pharmaceutical Research \& Manufacturers of America (PhRMA), which was involved in the preparation and revision of the manuscript. Dougherty is employed by PhRMA. Todor was a PQA-CVS Health Foundation Scholar who was funded to work on this study. Hines is employed by Pharmacy Quality Alliance. Wang reports grants from AbbVie, Curo, Bristol Myers Squibb, and Pfizer, during the time of this study, and fees from the PhRMA Foundation for work on its Heath Outcomes Research Advisor Committee. The other authors have nothing to disclose.

This study was presented as a poster at the online 2020 PQA Annual Meeting, May 7, 2020.

\section{ACKNOWLEDGMENTS}

The authors acknowledge the research assistance provided by Meagan Nicole Thornton and Ngoc Nguyen, both PharmD students at the corresponding author's institution.

\section{REFERENCES}

1. Centers for Medicare \& Medicaid Services (CMS), HHS. Medicare program; Medicare prescription drug benefit. Final rule. Fed Regist. 2005;70(18):4193-585.

2. Park T, Jung J. The effect of Medicare Part D on prescription drug spending and health care use: 6 years of follow-up, 2007-2012. J Manag Care Spec Pharm. 2017;23(1):5-12. doi: 10.18553/ jmcp.2017.23.1.5

3. Li R, Gregg EW, Barker LE, et al. Medicare Part D is associated with reducing the financial burden of health care services in Medicare beneficiaries with diagnosed diabetes. Med Care. 2013;51(10):888-93.

4. Choi YJ, Jia H, Gross T, Weinger K, Stone PW, Smaldone AM. The impact of Medicare Part D on the proportion of outof-pocket prescription drug costs among older adults with diabetes. Diabetes Care. 2017;40(4):502-08.
5. Medicare. Lower prescription drug costs. Accessed October 11, 2020. https://www.medicare.gov/yourmedicare-costs/get-help-paying-costs/ lower-prescription-costs

6. New York State Office for the Aging. Module 6: Medicare Prescription Drug Coverage. 2020 HIICAP

Notebook. Accessed July 2, 2021. https://aging.ny.gov/system/files/ documents/2020/08/medicare-prescription-drug-coverage.pdf

7. Chou YT, Farley JF, Stinchcombe TE, Proctor AE, Lafata JE, Dusetzina SB. The association between Medicare lowincome subsidy and anticancer treatment uptake in advanced lung cancer. J Natl Cancer Inst. 2020;112(6):djz183.

8. Biggers A, Shi Y, Charlson J, et al. Medicare D subsidies and racial disparities in persistence and adherence with hormonal therapy. J Clin Oncol. 2016;34(36):4398-404.

9. Yala SM, Duru OK, Ettner SL, Turk N, Mangione CM, Brown AF. Patterns of prescription drug expenditures and medication adherence among Medicare Part D beneficiaries with and without the lowincome supplement. BMC Health Serv Res. 2014;14:665.

10. Couto JE, Panchal JM, Lal LS, et al. Geographic variation in medication adherence in commercial and Medicare Part D populations. J Manag Care Pharm. 2014;20(8):834-42. doi: 10.18553/ jmcp.2014.20.8.834

11. Centers for Medicare \& Medicaid Services. Chartbook and charts. 2019. Accessed October 11, 2020. https://www. cms.gov/Research-Statistics-Data-andSystems/Statistics-Trends-and-Reports/ Chronic-Conditions/Chartbook_Charts

12. Research Data Assistance Center. Data file search. Accessed October 11, 2020. https://www.resdac.org/cms-data

13. Health Resources \& Services Administration. Area Health Resources Files. 2019. Accessed October 11, 2020. https://data.hrsa.gov/topics/ health-workforce/ahrf
14. Gelberg L, Andersen RM, Leake BD. The behavioral model for vulnerable populations: application to medical care use and outcomes for homeless people. Health Serv Res. 2000;34(6):1273-302.

15. Centers for Medicare \& Medicaid Services. Part D payment and risk adjustment. 2013. Accessed October 11, 2020. https://www.cms.gov/ Medicare/Prescription-DrugCoverage/DrugCoverageClaimsData/ RxClaims_PaymentRiskAdjustment

16. Jarrín OF, Nyandege AN, Grafova IB, Dong $\mathrm{X}$, Lin $\mathrm{H}$. Validity of race and ethnicity codes in Medicare administrative data compared with gold-standard self-reported race collected during routine home health care visits. Med Care. 2020;58(1):e1-e8.

17. Pharmacy Quality Alliance. Website. Accessed October 11, 2020. https://www. pqaalliance.org

18. Congressional Budget Office. Offsetting effects of prescription drug use on Medicare's spending for medical services. November 29, 2012. Accessed October 11, 2020. https://www.cbo.gov/ publication/43741

19. Col N, Fanale JE, Kronholm P. The role of medication noncompliance and adverse drug reactions in hospitalizations of the elderly. Arch Intern Med. 1990;150(4):841-45.

20. Task Force for Compliance. Noncompliance with medications: an economic tragedy with important implications for health care reform, 1994. Accessed July 2, 2021. https://www.npcnow.org/resources/ noncompliance-medications-economictragedy-important-implications-healthcare-reform-1994

21. New England Healthcare Institute. Thinking outside the pillbox: a systemwide approach to improving patient medication adherence for chronic disease. September 9, 2009. Accessed July 2, 2021. https://psnet.ahrq.gov/issue/ thinking-outside-pillbox-system-wideapproach-improving-patient-medicationadherence-chronic 
22. Briesacher BA, Gurwitz JH, Soumerai SB. Patients at-risk for costrelated medication nonadherence: a review of the literature. J Gen Intern Med. 2007;22(6):864-71.

23. Hughes CM. Medication nonadherence in the elderly: how big is the problem? Drugs Aging. 2004;21(12):793-811.

24. Institute of Medicine. Unequal Treatment: Confronting Racial and Ethnic Disparities in Health Care. The National Academies Press; 2003.

25. Keppel K, Bilheimer L, Gurley L. Improving population health and reducing health care disparities. Health Aff (Millwood). 2007;26(5):1281-92.

26. Phelan JC, Link BG, Tehranifar P. Social conditions as fundamental causes of health inequalities: theory, evidence, and policy implications. J Health Soc Behav. 2010;51(Suppl):S28-40.
27. Desai V, Nau D, Conklin M, Heaton PC. Impact of environmental factors on differences in quality of medication use: an insight for the Medicare Star Rating system. J Manag Care Spec Pharm. 2016;22(7):779-86. doi: 10.18553/ jmcp.2016.22.7.779

28. Cubanski J, Neuman T, Damico A, Smith K. Medicare beneficiaries' outof-pocket health care spending as a share of income now and projections for the future. January 26, 2018. Accessed October 11, 2020. https://www.kff.org/ report-section/medicare-beneficiariesout-of-pocket-health-care-spending-asa-share-of-income-now-and-projectionsfor-the-future-report/

29. Fidelity. How to plan for rising health care costs. 2021. Accessed July 2, 2021. https://www.fidelity. com/viewpoints/personal-finance/ plan-for-rising-health-care-costs
30. Briesacher BA, Ross-Degnan D Wagner AK, et al. Out-of-pocket burden of health care spending and the adequacy of the Medicare Part D low-income subsidy. Med Care. 2010;48(6):503-09.

31. Schoen C, Buttorff C, Andersen M, Davis K. Policy options to expand Medicare's low-income provisions to improve access and affordability. Health Aff (Millwood). 2015:34(12):2086-94.

32. Shoemaker JS, Davidoff AJ, Stuart B, Zuckerman IH, Onukwugha E, Powers C. Eligibility and take-up of the Medicare Part D low-income subsidy. Inquiry. 2012;49(3):214-30.

33. Decarolis F. The unintended effects of the Medicare Part D low income subsidy. Health Policy. 2015;119(5):597-603.

34. Duru OK, Edgington S, Mangione C, et al. Association of Medicare Part D lowincome cost subsidy program enrollment with increased fill adherence to clopidogrel after coronary stent placement. Pharmacotherapy. 2014;34(12):1230-38. 